\title{
Intra-Group Tension under Inter-group Conflict: A Generative Model using Group Social Norms and Identity
}

\author{
Roger M Whitaker ${ }^{1, *}$, Liam Turner $^{1}$, Gualtiero Colombo ${ }^{1}$, Dinesh Verma $^{2}$, \\ Diane Felmlee $^{3}$, Gavin Pearson ${ }^{4}$ \\ ${ }^{1}$ School of Computer Science \& Informatics, Cardiff University, \\ 5 The Parade, Cardiff, CF24 3AA, UK \\ ${ }^{2}$ IBM T.J. Watson Research Center, 1101 Kitchawan Road, \\ Yorktown Heights, NY, USA 10598 \\ ${ }^{3}$ Dept. of Sociology \& Criminology, Pennsylvania State University, \\ PA, USA 16802 \\ ${ }^{4}$ Defence Science \& Technology Laboratory, Porton Down, Salisbury, \\ Wiltshire, U.K, SP4 0JQ \\ \{WhitakerRM, TurnerL9, ColomboG\}@cardiff.ac.uk,dverma@us.ibm.com, \\ dhf12@psu.edu,agpearson@mail.dstl.gov.uk
}

\begin{abstract}
Group behavior is an important feature of conflict scenarios. Often such groups are chaotically organized, but their ideals are sociologically embedded across members such that the group has expected behavior that can represent a major threat. Therefore being able to model the evolution of groups on a generative basis, to anticipate their possible mutation, is valuable. However this is complex due to the diverse nature of human behavior and scenarios. In this paper we present an innovative approach to modeling these issues. Group identities are represented in terms of the behaviors (social norms) that members are expected to carry out towards other groups. Individuals predominantly compose their identity from the identity of the groups to which they belong, which is known to occur in situations of heightened conflict. The model introduced enables exploration of tensions associated with affiliation to multiple groups and the influence on inclusion and exclusion of individuals.
\end{abstract}

Keywords: Groups $\cdot$ Conflict $\cdot$ Social Norms $\cdot$ Identity $\cdot$ Generative modeling

\section{Introduction}

Understanding group behavior is highly valuable in situations of conflict and political instability. Over the years numerous significant theoretical insights have been made $[1,2,3]$, but particularly profound contributions have originated from Social Identity Theory [4]. A person's social identity is broadly a perception of who they are [5], and

* Corresponding author. 
in situations of heightened tension, the identity provided by a membership of a particular group can take precedence over the individual's sense of self (i.e., ones identity in the absence of any group). As a consequence of a heightened sense of belonging to a group, an individual naturally become disposed to adopting the beliefs and behavioral expectations, or social norms, of the group. Recent contributions have sought to develop this direction further, with Identity Fusion Theory [6] addressing the interplay between group and individual identities.

From these and other works, social identity has established itself as an important concept through which we can better understand human groups. In this paper, we use Social Identity Theory to develop a new and generative model of group behavior, specifically focusing on the tensions associated with affiliation to multiple groups. Our approach explores the natural pressures that exist between groups and the influence on inclusion and exclusion of individuals. We emphasize that our model does not seek to predict human behavior, but it exposes the potential tensions upon individuals when groups have conflicting expectations of the behavior of their members. This allows us to explore how group members become forced to migrate between groups, or become embedded within the social norms of a group. The use of simulation in this manner has become an important tool for identifying latent forces that shape behavior (e.g., [7]).

Our contribution also adds to the state-of-the-art in generative modeling of group behavior $[8,9]$ by providing a simple but effective event driven approach. The stateof-the-art in this area is at a formative stage, with numerous interesting approaches now emerging (e.g., [10]). The key distinctive feature of our model is the assumed prevalence of groups and their influence on the individual: specifically each individual is represented as a combination of the identities of the groups to which they belong. This is motivated by Social Identity Theory and contrasts with a conventional modeling approach where individuals are the dominant modeling element, from which groups are then defined based on a collection of common characteristics.

A further advantage of our approach is that it provides a flexible framework for controlling and extending the number of parameters in the model, which enhances prospects for useful experimentation. This is an important element in generative modeling for social computation, particularly from the user's perspective, as it reduces the extent of a-priori learning required to understand parameter sensitivity and parameter interaction. This supports the exploration of groups, including the prospects for intelligent forecasting of changes to their size and individual affiliations.

\section{$2 \quad$ Model}

The model we adopt has simple underlying principles. A population of individuals and a set of groups represent the main components. Individuals, or agents, are affiliated with multiple groups, dividing their commitment (i.e., time and resources) across their group affiliations. This is modeled by each individual having a weighting vector $\left(w_{1}, w_{2}, \ldots w_{\mathrm{n}}\right)$ where $w_{\mathrm{i}}$ indicates the proportion of the agent's commitment to group $G_{\mathrm{i}}\left(\sum_{i=1}^{n} w_{i}=1\right)$. The weighting vector signals the groups to which an individual is affiliated and for demonstration purposes we use a population of 100 agents and four groups. Individuals are randomly assigned to groups at the beginning of the simula- 
tion. In all experiments, $G_{0}$ initially has 73 members, $G_{1}$ and $G_{2}$ have 78 , and $G_{3}$ has 76 members.

\subsection{Representing group}

Each group has an identity that is described by the degree to which its members should perform different actions towards the other groups. Table 1 shows the five types of action that have been chosen for demonstration purposes. Each action is described by a cost to the individual and an impact (positive or negative) on the receiving group. This framework allows extreme as well as mainstream out-group behavior to be modeled. Table 2 shows how we represent a group's expected norms, defining the expected frequency of different actions, which we call a social norm vector. A group may apply different expectations of behavior to different groups: Table 3 demonstrates this using our four example groups.

\subsection{Representing the individual}

Although individuals act on behalf of the groups that they are affiliated with, we also make provision for individuals to possess their own individual identity. This is a social norm vector (Table 2) that they will choose to identify with in the absence of affiliation with any group. We assume the individual social norm as the mainstream behaviour $S N_{1}$ for $75 \%$ of the population, $S N_{2}$ for 20 , and $S N_{3}$ for the remaining $5 \%$. We also invoke an individual compliance parameter for each agent $j$, denoted comp $_{j}$, which governs the chance that $j$ complies with a group's norm, rather than its own norm, when conducting an action on behalf of a group (Section 2.3). This is set as $95 \%$ chance for all individuals.

\subsection{Individuals perform actions on behalf of a group towards other groups}

The simulation applies a sequence of 100 rounds, after which an evolutionary step is applied (Sections $2.4,2.5$ ), creating the next generation and after which a new sequence of rounds occurs. For this demonstration, 100 generations are performed and individuals have 100 units of resource available for performing actions at the start of each generation.

In each round, each individual $j$ randomly selects a group to act for, from the groups to which $j$ belongs (determined as $i$ for which $w_{i}$ is $>0$ ), and performs an action towards another group if their current associated resources is equal or greater to the chosen action's cost. The group that $j$ chooses to act for, say $G$, is randomly chosen but weighted by $j$ 's weighting vector. At the same time, a target out-group, say $G^{*}$, is randomly selected for the action, where $G$ and $G^{*}$ are distinct. Note that $j$ may also be a member of $G^{*}$. If $j$ is not a member of any group (due to previous exclusion) they are considered independent and can continue to act, and effectively constitute their own group.

The action that $j$ undertakes is defined by the social norms of $G$ in respect of $G^{*}$

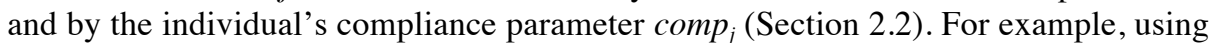
Table 3, if $G=G_{1}$, and $G^{*}=G_{0}$, then $j$ selects an action on a random basis weighted 
by the social norm vector $\mathrm{SN}_{2}$, as defined in Table 2, which overall represents negative behavior. Whether the individual performs the action is determined randomly using $\operatorname{comp}_{j}$, in which there is a $1-\operatorname{comp}_{j}(5 \%)$ chance than an individual reverts to a action with the probability of selection weighted by its own norm (Section 2.2). If a user is not affiliated with a group then the action towards $G^{*}$ is chosen from the individuals own norm.

When an action is performed, $j$ uses their associated resources defined in Table 1. At the same time the cumulative impact on group $G^{*}$ is recorded, as defined by the schedule in Table 1. For demonstration purposes we perform 100 rounds before evolution is applied (Sections 2.4 and 2.5).

\subsection{Evolution and exclusion from a group}

After the sequence of 100 rounds has been completed (Section 2.3), an evolutionary step is performed. This step looks at the overall actions that individuals have performed against the expectations of the groups to which they are affiliated, and considers whether they are candidates to be excluded. This is carried out using processes under two themes: (i) the tolerance that a group applies with respect to impact on the group by its own members while they are acting on behalf of other groups; (ii) the normativity of group, representing the strictness with which group members should perform actions on other groups consistent with expected social norms.

The tolerance and normativity are attributes that characterize the nature of a group, alongside the group's social norm vectors. For example, extreme groups would be expected to exhibit high levels of normativity, while liberal groups would be the opposite. Additionally, defining tolerance and normativity allows us to characterize groups based on their in-group/out-group disposition, since tolerance is focused on ingroup expectations while the normativity is focused on out-group expectations. Our definition of normativity is inspired by its sociological definition [11], representing the extent to which members should feel that they ought to behave in a particular way.

For exclusion based on intolerance, assuming that $j$ is a member of $G$, if the cumulative negative impact that $j$ has performed towards $G$ within a generation is beyond the value of $e x-$ tol $_{G}$ then $j$ is flagged for removal from $G$. This can occur if $j$ acts negatively towards $G$ when representing another group. The value of $e x-$ tol $_{G}$ for all groups $G$ is defined in Table 4.

For exclusion based on deviation from normativity, each group $G$ considers the percentage of positive impact verses negative impact that each individual makes towards all other groups after 100 rounds. This is compared with their own social norms towards other groups. The exclusion strictness, ex-strict ${ }_{G}$, is the parameter used to determine how much an individual can deviate from $G$ 's expectations of out-group behavior. If an individual deviates in their out-group behavior towards all groups and is a member of the $G$, they will be flagged for exclusion.

Using $G_{0}$ as an example, the expected impact of the social norm applied by $G_{0}$ toward other groups is calculated and used for comparison with group member $j$. We use $G_{0}$ as defined in Table 3, where $G_{0}$ is an extreme group applying $S N_{3}$ (Table 2) to all other groups. Starting with $G_{1}$, only $1 \%$ of actions from $G_{0}$ are expected to be positive leading to an expected positive impact of just 0.1 units. $40 \%$ of expected actions are negative and $10 \%$ of expected actions are strongly negative, resulting in an ex- 
pected negative contribution of -54 units. This means that $0.18 \%$ of total impact on $G_{1}$ is expected to be positive and $99.82 \%$ of impact is expected to be negative.

These values are similarly computed in respect of $G_{2}$ and $G_{3}$, and compared to the negative/positive impact made by $j$ towards $G_{1}, G_{2}$, and $G_{3}$ during the previous 100 rounds. If at least one of these are within ex-strict ${ }_{G I}$ percent of the expected positive/negative impact on each group (i.e., $0.18 \%$ and $99.82 \%$ respectively for this example) then $j$ remains in $G_{0}$, otherwise $j$ is flagged for exclusion from $G_{0}$. This process is then repeated for $G_{1}, G_{2}$, and $G_{3}$.

After considering all individuals in the population, those that are flagged to be excluded from each of the groups are removed by setting their commitment weighting $(w)$ to zero for the these groups. For such individuals, we re-normalize the non-zero elements of the weighting vector $\left(w_{1}, w_{2}, \ldots w_{\mathrm{n}}\right)$ to ensure that $\sum_{i=1}^{n} w_{i}=1$ once again. This represents the agent increasing their commitment to the groups that they remain affiliated with, while preserving the ratio of time spent between them.

\subsection{Evolution and admission to a group}

The concepts of tolerance and normativity can also be used to model admission to a group, analogous to the processes for exclusion. We use the threshold, denoted in-tol ${ }_{G}$, to govern the admission of $j$ to a group $G$ (shown in Table 4). The individual $j$ must have made a positive impact to $G$, which is at least $i n$-tol $l_{G}$ in magnitude, over the previous 100 rounds. A threshold in-strict $_{G}$ is used to govern admission based on normativity (shown in Table 4), adopting a process that works analogous to that for exclusion based on normativity; with the exception that for an individual to be flagged for inclusion into a given group $G$, their impact towards all other groups must be within in-strict ${ }_{G}$ percent.

Using these processes individuals are flagged for potential inclusion to particular groups, joining with a weight $w$ of 0.1 . Additionally, there is a small chance that an individual will join a group with a weight of 0.1 through mutation, set at a probability $p_{\text {join }}$ of 0.01 for testing purposes for all groups. Mutation represents serendipity and random changes in behavior or scenarios. For each individual $j$, to accommodate the commitment to new groups, we renormalize the existing group weights, while preserving the ratio of time spent between pre-existing group affiliations, to ensure that $\sum_{i=1}^{n} w_{i}=1$.

\subsection{Presentation of the Model}

Example pseudo-code summarising the evolutionary steps.

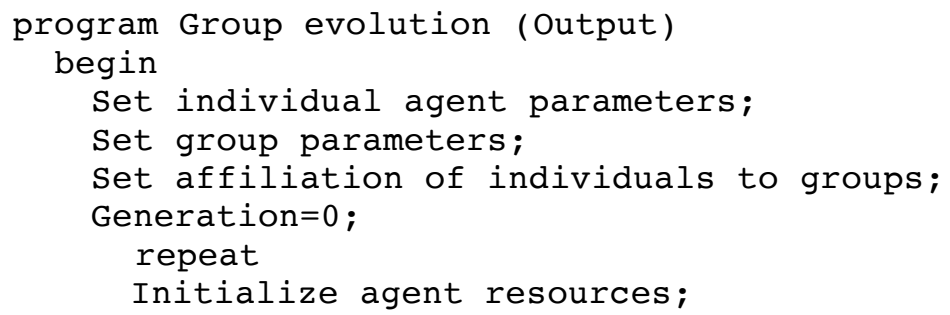




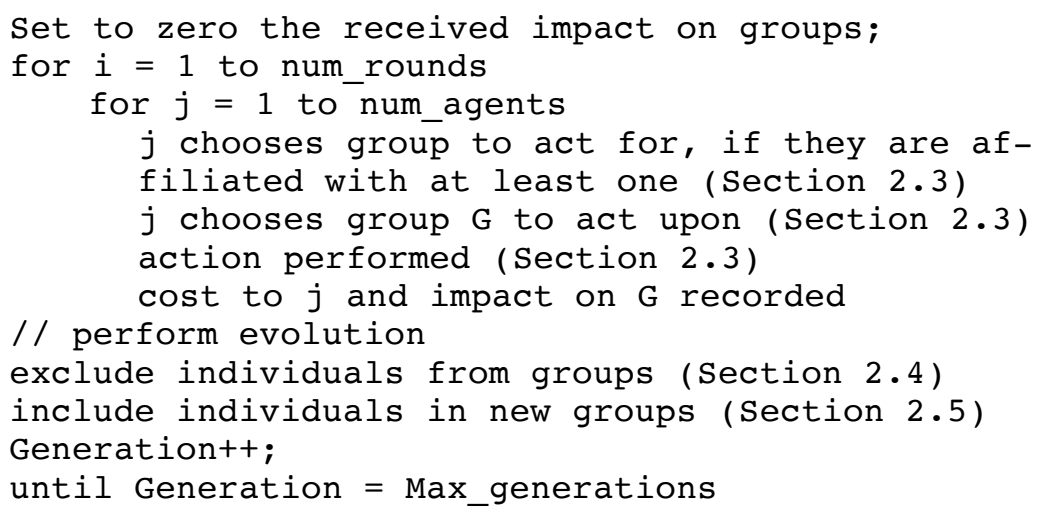

Above we summarize the overall pseudo-code that represents the simulation framework. Monitoring the weighting vectors throughout the simulation allows group size and shape to be assessed. We set Max_generations to be 100, num_rounds to be 100 , num_agents to be 100 , and resources to be 100 .

\section{Scenarios}

We adopt a scenario of four groups as described in Tables 3 and 4, which documents how each of the four groups expect their members to behave towards other groups, and how tolerable they are to in-group impact and strict towards out-group impact. The social norm vectors defined in Table 2 determine these behaviors. In turn, the social norm vectors are specified by the actions in Table 1 . In the scenario, $G_{0}$ is an extremist group that is strongly negative to all other groups, while the others (i.e., $G_{1}$, $G_{2}, G_{3}$ ) are negative towards $G_{0}$, but not extreme, and positive towards each other.

We note that the format of input in Tables 1, 2 and 3 means that interesting and complex scenarios can be defined and examined. The range of action types (Table 1) is extensible and can be specified to include a wide range of potential actions that are specific to a particular group or scenario.

Table 1. Specification of actions that individuals may perform in terms of cost to the individual and impact on the receiving group.

\begin{tabular}{llll}
\hline Action Type & Description & Cost to individual & Impact on the receiving group \\
\hline 1 & Strong positive action & 100 & 500 \\
2 & Positive action & 5 & 10 \\
3 & Indifferent action & 1 & 0 \\
4 & Negative action & 5 & -10 \\
5 & Strong negative action & 100 & -500 \\
\hline
\end{tabular}


Table 2. Social norm vectors used in the scenarios by groups and individuals, describing the mean frequency of actions types.

\begin{tabular}{lllllll}
\hline $\begin{array}{l}\text { Social } \\
\text { norm } \\
\text { vector }\end{array}$ & Description & $\begin{array}{l}\text { Action } \\
\text { type 1 } \\
\text { (Strong +ve) }\end{array}$ & $\begin{array}{l}\text { Action } \\
\text { type 2 } \\
\text { (+ve) }\end{array}$ & $\begin{array}{l}\text { Action } \\
\text { type 3 } \\
\text { (Neutral) }\end{array}$ & $\begin{array}{l}\text { Action } \\
\text { type 4 } \\
\text { (-ve) }\end{array}$ & $\begin{array}{l}\text { Action } \\
\text { type 5 } \\
\text { (Strong -ve) }\end{array}$ \\
\hline$S N_{1}$ & Mainstream & $5 \%$ & $60 \%$ & $34 \%$ & $0.9 \%$ & $0.1 \%$ \\
$S N_{2}$ & Negative & $0 \%$ & $10 \%$ & $75 \%$ & $14.9 \%$ & $0.1 \%$ \\
$S N_{3}$ & Extremist & $0 \%$ & $1 \%$ & $49 \%$ & $40 \%$ & $10 \%$ \\
\hline
\end{tabular}

Table 3. Scenario specification: three groups are mostly mainstream $\left(G_{1}, G_{2}, G_{3}\right)$, and one is extremist $\left(G_{0}\right)$.

\begin{tabular}{lllll}
\hline & \multicolumn{4}{l}{ Social Norm Vector applied to group: } \\
\hline Group & $G_{0}$ & $G_{1}$ & $G_{2}$ & $G_{3}$ \\
\hline$G_{0}$ & & $S N_{3}$ & $S N_{3}$ & $S N_{3}$ \\
$G_{1}$ & $S N_{2}$ & & $S N_{1}$ & $S N_{1}$ \\
$G_{2}$ & $S N_{2}$ & $S N_{1}$ & & $S N_{1}$ \\
$G_{3}$ & $S N_{2}$ & $S N_{1}$ & $S N_{1}$ & \\
\hline
\end{tabular}

Table 4. Scenario specification: normativity (i.e., strictness) and tolerance for the four groups.

\begin{tabular}{lllll}
\hline Group & in-strict $_{G}$ & ex-strict & in-tol & ex-tol \\
\hline$G_{0}$ & .1 & .1 & 50 & -50 \\
$G_{1}$ & .5 & .5 & 50 & -50 \\
$G_{2}$ & .5 & .5 & 50 & -50 \\
$G_{3}$ & .5 & .5 & 50 & -50 \\
\hline
\end{tabular}

\section{$4 \quad$ Results}

In this section we present proof of concept results that show how different assumptions affect the size of groups over time, and effects on multi-group affliation.

\subsection{Applying tolerance but not normativity allows extremist groups to dominate}

We begin by exploring how in-group tension can form. Fig 1 shows the sizes of the four groups after each generation when tolerance $\left(e x-t_{G} l_{G}\right.$ and $i n-t o l_{G}$, in Section 2.4 and 2.5 respectively) is the only measure used for inclusion and exclusion. Normativity criteria are not applied. In Fig 1 and similar plots the y axis is cumulative.

The results (Fig 1 and 2) show $G_{0}$ continuing to grow over time with the other groups shrinking. This occurs due to the heightened positivity in the social norms for $G_{1}, G_{2}$, and $G_{3}$, in comparison to $G_{0}$. Individuals perform generally positive actions towards $G_{0}$ when representing $G_{1}, G_{2}$, and $G_{3}$, presenting no barrier to inclusion to $G_{0}$. As the same time, individuals acting on behalf of $G_{0}$ perform negative actions towards the other groups, resulting in an increased chance of exclusion from $G_{1}, G_{2}$, and $G_{3}$. 


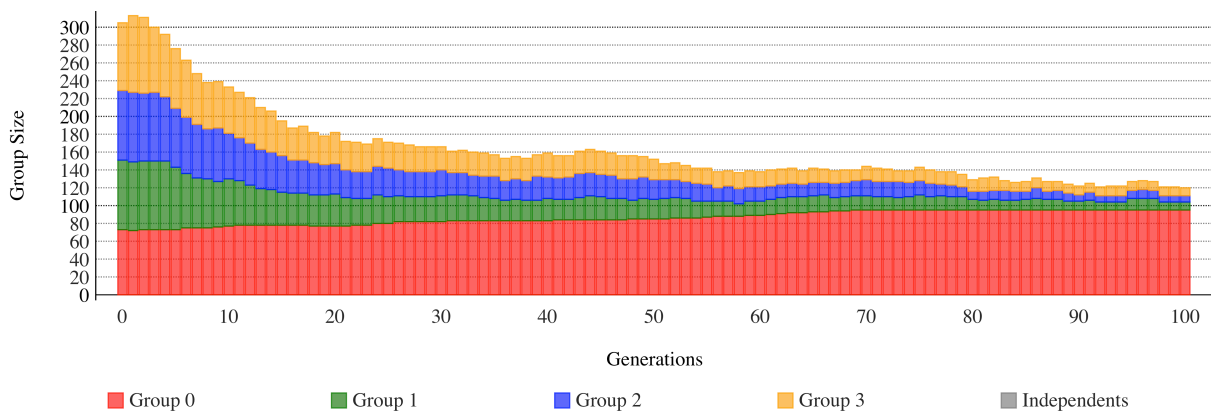

Fig. 1. Changes in group size based on groups only using tolerance but not normativity.

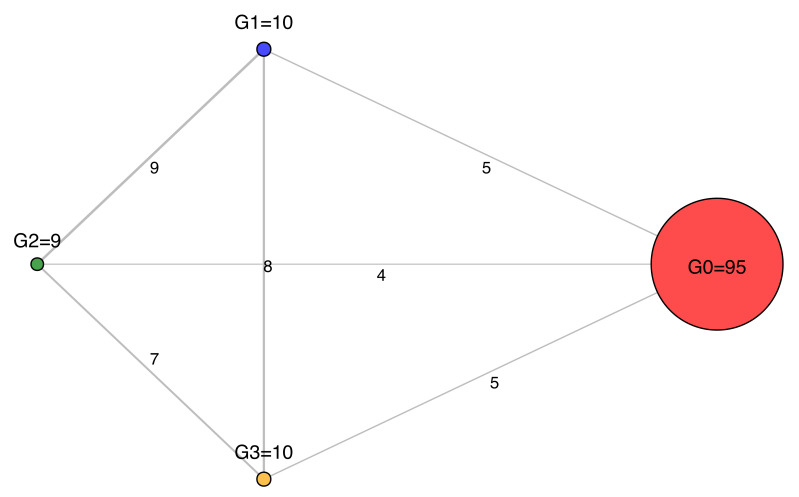

Fig. 2. Cross-group membership after generation 100 when only in-group tension is considered using $e x-$ tol $_{G}$ and $i n-$ tol $_{G}$. Nodes are scaled proportionally to the group they represent. Edges represent the size of the intersection in membership between two groups.

Additionally since exclusion mechanisms are only based on the negative impacts received, extremist groups tend not to expel members that may be acting according to the social norms of mainstream groups. Members of $G_{0}$ are not invited into mainstream groups as they very infrequently perform any positive actions.

This is evident in Fig 2, which shows the majority of members of $G_{0}$ cutting ties with the other groups by the time of generation 100 , except for a small percentage, who are sustained through a the combined effects of mutation (i.e., random incidences) and use of individual identity and their own social norm (5\% chance).

\subsection{Tolerance and normativity lead to group stability, and suppress dominance of the extremist group}

In contrast to Fig 1, Fig 2 displays the results when both tolerance ( $e x$-tol ${ }_{G}$ and intol $_{G}$ ) and normativity (ex-strict ${ }_{G}$ and in-strict $_{G}$, in Section 2.4 and 2.5 respectively) are applied in the inclusion and exclusion processes. The results show that considering how members act towards other groups impedes $G_{0}$ from growing. All groups co-exist over time with relative stability, which is without any one single group dominating. 


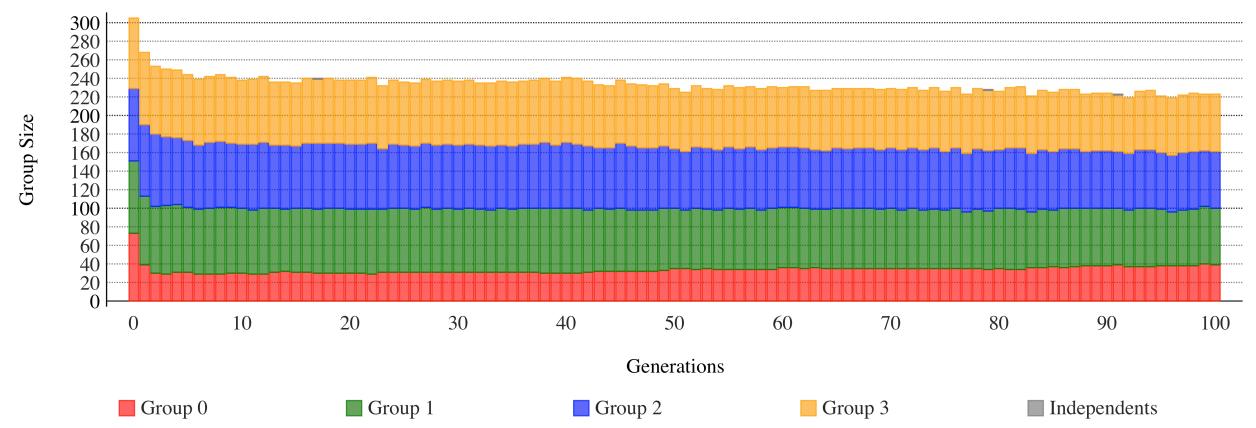

Fig. 3. Changes in group size based on groups only using tolerance and normativity
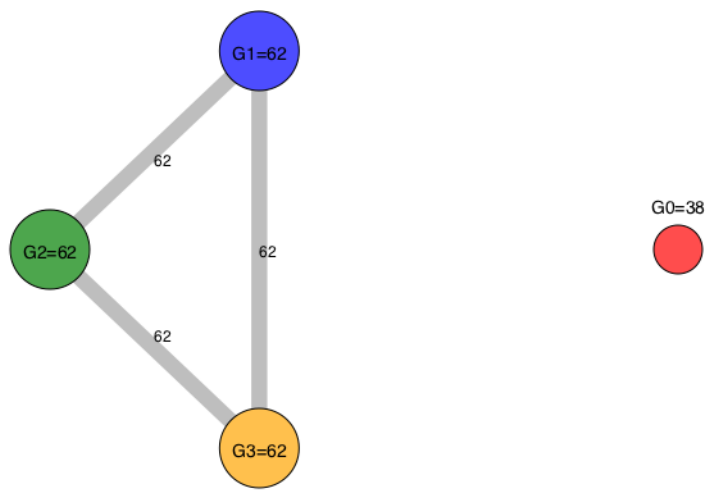

Fig. 4. Cross-group membership after generation 100 when both tolerance (ex-strict $t_{G}$ and instrict $_{G}$ and normativity (ex-strict ${ }_{G}$ and in-strict $_{G}$ ) are considered. Nodes are groups sized proportionally with the number of members and edges represent the size of the intersection in membership between two groups.

The greater number of mainstream groups means that there is a greater chance of individuals acting towards $G_{0}$ 's out-groups in a manner that it deems incompatible to expectations. This reaffirms the presence of the groups $G_{1}, G_{2}$, and $G_{3}$. It also indicates that the number of groups, as well as their size, impacts on the dynamics and this is worthy of further investigation.

This is also reflected through individuals in $G_{0}$ cutting ties with other groups, with Fig 4 showing all members of $G_{0}$ cutting ties by generation 100 (a small number occasionally re-appear for a short period through the subsequent generations, due to mutation). In contrast, members of the mainstream groups are more capable of maintaining strong ties among each other and this reaches a $100 \%$ level of shared membership after a relative short number of generations.

It is also worth commenting that during the course of this investigation, we experimented with the extent of the strictness parameter for normativity, particularly for $G_{0}$. Under definition of the parameter, increasing the parameter increases leniency, potentially increasing the pool of individuals that can be admitted to $G_{0}$. We find that due to the extreme social norms of $G_{0}$, high levels of leniency (e.g., >.98) are required to substantially increase the size of $G_{0}$. This destabilizes the scenario allowing $G_{0}$ to 


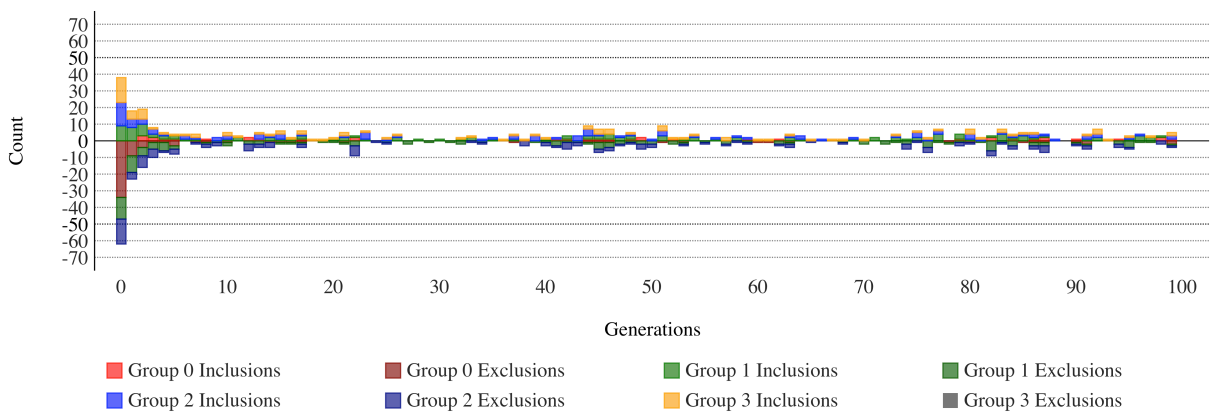

Fig. 5. Changes in group size based on groups only using tolerance and normativity

dominate. This also reflects the potentially crude nature of the judgment process used to model normativity.

In Fig 5 we show the number of inclusions and exclusions that occur over course of the simulation. The results show high levels of changes to initial membership, which is due to the random allocation of group membership at the beginning. However, after around 10 generations this reduces and any events predominantly due to mutation or the resulting change in behavior due to mutation (discussed in Section 2.5). This supports the findings in Fig 4: under these conditions the population quickly divides between positive and negative norms.

A final observation is that some individuals occasionally get excluded from all groups and become independent. However, this is unsurprisingly short-lived (as shown in Fig 3), because when this happens individuals revert to their own social norms, which for this scenario, align closely with those used in at least one group.

\subsection{Prohibiting re-admission to groups after exclusion marginally affects mainstream groups more than the extremist group}

Fig 6 shows the effect of prohibiting re-admission to each group after their first exclusion. Once individuals become affiliated in the extremist group, their affiliation with other (mainstream) groups is severed (Fig 4). This is occurs as a consequence of the mainstream groups executing exclusion. As extremist group members are more likely to be isolated to that group, there is much less a likelihood of them acting on behalf of other groups in an incompatible way.

As a result we observe that the extremist group $G_{0}$ tends to remain relatively static in size while the mainstream groups tend to marginally decline in membership (Fig 6). At the same time the number of individuals without affiliation to a group grows. 


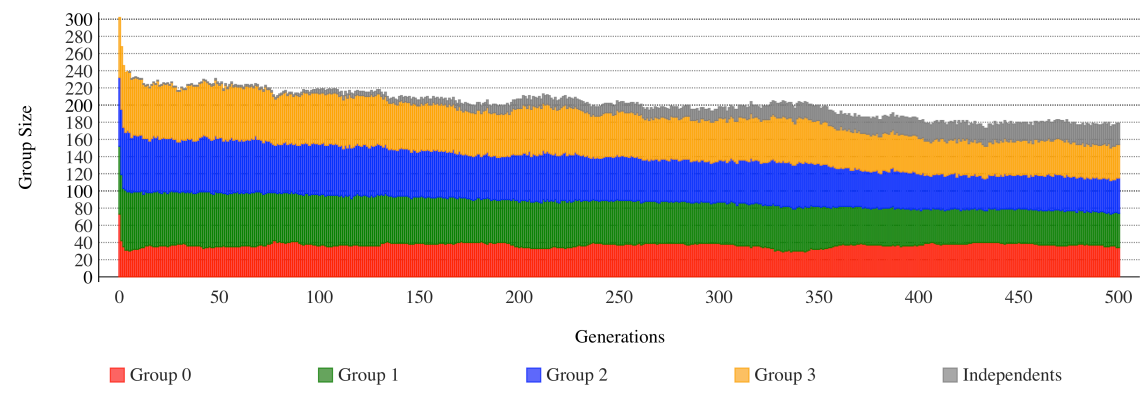

Fig. 6. Group size over 500 generations when re-admission to groups is prohibited.

\section{Conclusions}

The results demonstrate a first proof of concept for a model concerning the tensions on individuals that belong to multiple groups where expectations on behavior differ. This is a new approach to modeling groups that allows us to assess the changes in a group's size as a consequence of behavioral social norms of groups and the affiliation of individuals between them. The model represents a group's identity behavioral expectations (social norms), which can also be included for individuals.

The model allows observation of the natural tensions that exist between belonging to multiple groups when their social norms differ. Consequently we can use the model to gain insight into the features that influence the evolution of particular types of groups, such as those with extremist tendencies.

From testing, the initial results exemplify conditions that affect the stability and growth of groups. In particular, the judgment processes that groups apply for inclusion and exclusion have a significant effect. Extreme groups prosper, in terms of recruitment, when they are in-group focused, making judgments only based on the extent of negative actions done towards them by individuals. As soon as extreme groups judge potential members based on their actions towards the out-groups, this restricts admission and their subsequent growth, unless there is extreme leniency. This reaffirms that recruitment strategies for extreme groups may masquerade in mainstream forms to gain traction.

The results also show that individuals can successfully coexist in mainstream groups while membership of an extremist group requires that individuals sever their affiliations with other groups. Numbers of groups and their relative similarity also appear to influence potential growth of groups, and this warrants further exploration.

\subsection{Future Work}

In summary, the model represents an interesting framework for considering group dynamics based on the natural tensions that exist between behavioural expectations. It can be extended and refined in a number of directions to include group-based scenarios for wide ranging situations, and with additional levels of detail. This development can also include modeling interventions and assessing their effects, with calibration of 
parameters based on real world scenarios. Further contextual factors, individual differences and group effects can potentially be included. These will support future generative modeling capability, which is currently at a formative stage.

\section{Acknowledgement}

This research was sponsored by the U.S. Army Research Laboratory and the U.K. Ministry of Defence under Agreement Number W911NF-16-3-0001. The views and conclusions contained in this document are those of the authors and should not be interpreted as representing the official policies, either expressed or implied, of the U.S. Army Research Laboratory, the U.S. Government, the U.K. Ministry of Defence or the U.K. Government. The U.S. and U.K. Governments are authorized to reproduce and distribute reprints for Government purposes notwithstanding any copyright notation hereon.

\section{References}

1. Pettigrew TF, Tropp LR. A meta-analytic test of intergroup contact theory. J. Personality and Social Psychology, 90(5):751 (2006)

2. Allport GW. The nature of prejudice. Basic books (1979)

3. Fiske ST. What we know now about bias and intergroup conflict, the problem of the century. Current Directions in Psychological Science. 1;11(4):123--8 (2002)

4. Tajfel H, Turner JC. An integrative theory of intergroup conflict. The social psychology of intergroup relations, 33(47):74 (1979)

5. Hogg MA. Social identity theory. In: Understanding Peace and Conflict Through Social Identity Theory pp. 3-17. Springer International Publishing (2016)

6. Swann Jr WB, Gómez Á, Seyle DC, Morales J, Huici C. Identity fusion: the interplay of personal and social identities in extreme group behavior. J. Personality and Social Psychology. 96(5):995 (2009)

7. Whitaker RM, Colombo GB, Allen SM, Dunbar RI. A dominant social comparison heuristic unites alternative mechanisms for the evolution of indirect reciprocity. Scientific Reports 6 (2016)

8. Whitaker RM, Felmlee D, Verma DC, Preece, A, Williams G. From evolution to revolution: understanding mutability in large and disruptive human groups. In SPIE Defense and Security, to appear (2017)

9. Verma DC, Verma Felmlee D, A, Pearson G, Whitaker RM. A generative model for predicting terrorist incidents. In SPIE Defense and Security, to appear (2017)

10.Naugle AB, Bernard ML. Using Computational Modeling to Examine Shifts Towards Extremist Behaviors in European Diaspora Communities. In: Advances in Cross-Cultural Decision Making, pp. 321-332. Springer International Publishing (2017)

11.Anderson E. Beyond homo economicus: New developments in theories of social norms. Philosophy \& Public Affairs. 1;29(2):170-200 (2000) 